\title{
Hutchinson-Guilford progeria syndrome
}

\author{
P K Sarkar, R A Shinton
}

Progeria is a human disease model of accelerated ageing. ${ }^{1-3}$ The progeria syndrome is a rare genetic disorder, first reported in 1886 by Hutchinson and Guilford in England. ${ }^{4}$ The inheritance pattern, paternal age effect, and lack of consanguinity argue that it is due to a sporadic dominant mutation. HutchinsonGuilford progeria syndrome (HGPS) is associated with several features of premature ageing - for example, growth retardation, characteristic facies, loss of hair, and subcutaneous fat, restricted joint mobility, prominent eyes, and severe premature atherosclerosis. ${ }^{5}$ The exact pathophysiology of this syndrome is not known. Recent findings in several laboratories indicate that progeria patients excrete an excessive amount of glycosaminoglycan, hyaluronic acid. ${ }^{67}$

In 1886, Jonathan Hutchinson reported a case of a 3.5 year old boy who had the appearance of an old man. ${ }^{4}$ Later, Hastings Guilford reported a second case with similar features. ${ }^{8}$ The term, "progeria" was taken from the Greek word for old age, "geras", 89 originally proposed by Guilford in 1904 .

The interesting clinical features consist of craniofacial disproportion, micrognathia, prominent scalp veins, scalp alopecia, prominent eyes, wrinkled skin, protruding ears, nail dystrophy, midfacial cyanosis, growth retardation, and a sculpted nose at birth. DeBusk observed that patients with progeria syndrome are typically considered normal infants. ${ }^{5}$ The characteristic facies, posture, stiffness of joints, and bone, teeth, and skin changes become apparent during the second year of life. The necropsies on patients with HGPS revealed prominent abnormalities in the skin, cardiovascular tissues, and bone. ${ }^{9}$

In 1972, DeBusk presented case reports of four patients and reviewed the world literature on HGPS. Although these patients develop premature atherosclerosis and die of cardiac or cerebral vascular disease between 7 and 27 years of age, many other features associated with pathological ageing are absent. ${ }^{5}$

\section{City Hospital NHS Trust, Birmingham, UK \\ P K Sarkar \\ Birmingham \\ Heartlands Hospital, Birmingham, UK R A Shinton \\ Correspondence to: Dr P K Sarkar, Selly Oak Hospital, Raddlebarn Road, Birmingham B29 6JD, UK}

Submitted 19 June 2000 Accepted 29 August 2000

\section{Epidemiology}

The estimated incidence of HGPS in the USA is one in eight million births, based on the number of cases. Brown suggested that only one half of the affected patients were reported, thereby the estimated incidence of one in four million live births. ${ }^{3}$ Males are affected one and a half times more often than females ( $M$ : $F=1.5: 1)$. Ninety seven per cent of affected patients are white. ${ }^{59}$ Several rare conditions exist in human beings that exhibit certain phenotypic characteristics associated with senescence. Often referred to as "segmental progeroid syndromes", the most important and widely studied condition was HGPS. ${ }^{10}$
The exact inheritance of HGPS is not known. In the past, it was thought to be an autosomal recessive. DeBusk reviewed only three families where more than one member was affected, consanguinity was uncommon, and advanced paternal age was noted. ${ }^{5}$ These observations made an autosomal recessive inheritance very unlikely and favour a sporadic, dominant mutation. Since most cases are due to isolated mutations of a gamete, the familial occurrence is rare. ${ }^{911}$ Some cases may be due to germ line mutations, and therefore, parents of one affected child should be counselled that the risk of a subsequent affected child is one in 500 for each pregnancy. ${ }^{11}$

In Europe, 26 cases of premature ageing syndromes (for example, progeria, Werner's syndrome) were reported between 1995 and 1996 by the Eurostat (social and regional statistics and geographical information system of Europe), Luxembourg-Kirchberg. Progeria syndrome is coded under "other endocrine disorders" which also include Werner's syndrome and pineal gland dysfunction according to the International Classification of Diseases, ninth revision (ICD-9) 259.8. ${ }^{12}$ Life spans of various premature ageing syndromes are different, as for example, fifth/sixth decades for Werner's syndrome, second/third decades for progeria syndromes and so on. ${ }^{513}$ In Europe, out of 26 cases of premature ageing syndromes, eight patients died before the age of 25 years.

The authors contacted the Office for $\mathrm{Na}-$ tional Statistics (ONS), UK and Eurostat, Luxembourg-Kirchberg regarding progeria syndrome. Causes of death recorded on the death certificate were coded by ONS and Eurostat according to the ICD-9. ${ }^{12}$ There were only a handful of deaths between 1993 and 1998 in the UK and Europe where ICD-9 259.8 was given as the underlying cause of death or was mentioned as a contributory cause of death. Progeria syndrome was specifically mentioned on the death certificate in only five of these cases in the UK.

\section{Clinical features}

Patients with this condition generally appear normal at birth, but by the age 1 or 2 years severe growth retardation is usually observed. Generally, these children have short stature and lower weight for height, and usually have absent sexual maturation. Balding occurs due to the receding hair line and loss of eye brows and eye lashes is common in the early childhood. Skin is lax and wrinkled due to widespread loss of subcutaneous fat. There is also circumoral cyanosis (table 1).

Features in the head consist of craniofacial disproportion, micrognathia, alopecia, prominent scalp veins, prominent eyes, a beaked nose, and a "plucked bird" appearance. The 
large bald head and a small jaw give them an extremely aged appearance. They are usually short and thin with an average height of $100 \mathrm{~cm}$ or so and average weight of $12-15 \mathrm{~kg}$ or even less. Other clinical features include abnormal and delayed dentition, thin and high pitched voice, pyriform thorax, and short dystrophic clavicles.

The limbs are usually thin and may be associated with stiff joints, coxa valga, and dystrophic nails. They also demonstrate "horse riding" stance and wide based shuffling gait. The bones might show distinctive changes-for example, resorption of clavicles and replacement by fibrous tissue, resorption of terminal phallanges (acro-osteolysis), etc. Aseptic necrosis of the head of the femur and hip dislocation are also common. ${ }^{14} 15$ They have a normal IQ and mentation. The median age of death is 12 years. $^{3}$

\section{Laboratory, biochemical, and cellular abnormalities}

There are various studies that demonstrated the fundamental biochemical and cellular abnormalities in this syndrome. Normal human cells will not divide forever, even in culture. After a defined number of cell divisioins, every culture enters a viable non-dividing state termed senescence. ${ }^{16}$ This led to the proposal that the progressive accumulation of senescent cells contributes to (but does not exclusively cause) the ageing process, as described by Faragher and Kipling. ${ }^{16}{ }^{17}$ The eventual cessation of cell division is accompanied by a specific set of changes in cell physiology, morphology, and gene expression. Such changes in phenotype have the potential to contribute to human ageing and age related diseases. Recent data have demonstrated the presence of senescent cells in the aged human tissues. ${ }^{17}$ Substantial progress has been made in the last few years towards identifying the cell cycle regulatory mechanisms and the telomere ageing clock theory, executing senescence. ${ }^{18}$

The telomeres (the sections of DNA occurring at the end of chromosomes) consist of repeated groups of the base sequence TTAGGG, where $T, A$, and $G$ represent the bases thymine, adenine, and guanine, respectively. These telomeres' lengths are maintained by the activity of the enzyme, telomerase, and are thought to be important protective factors in maintaining the integrity of chromosomes. ${ }^{19}$ It now appears that in vitro replicative senescence, which has been observed in cultured somatic cells, is caused by a loss of telomere length in those cells, caused by inactivity of telomerase. ${ }^{19}$ Reintroduction of telomerase prevents the onset of senescence. This has led to the proposition that telomerase activity is an important determinant in human ageing. Human cells that do not proliferate frequently have now been shown to have active telomerase and therefore will not lose telomere length.

Studies of telomerase knock-out mice have demonstrated some aspects of accelerated ageing after three generations, but the relevance of these observations to normal ageing remains unconvincing. ${ }^{19}$ It raises the possibility that telomere length and telomerase activity may be important factors for the pathogenesis of premature ageing in HGPS. The observation that telomere length of somatic chromosomes progressively declines with increasing age and that progeria cells have short telomeres lends support to the idea of a cellular "clock", preventing further replication at a critically reduced length and therefore allowing DNA damage to occur unrepaired. ${ }^{18} 20$

In HGPS, the most important biochemical changes occur within the connective tissue, mainly of mesodermal origin. The most useful finding in this syndrome appears to be the urinary excretion of hyaluronic acid. There are at least two studies that demonstrated 10-20 times greater urinary excretion of hyaluronic acid in patients with HGPS than with controls. ${ }^{61}$ Hyaluronic acid is an unsulphated glycosaminoglycan that maintains the integrity and texture of the skeletal, muscular, cutaneous, and vascular systems. These hyaluronic acid abnormalities might explain sclerodermalike skin changes, collagen hardening, and calcification of arterial walls. ${ }^{22}$ There was experimental evidence that hyaluronic acid containing implants were shown to cause avascularity when implanted into normal vascular wing mesoderm. ${ }^{23}$

Hyaluronic acid, therefore, appears to be very crucial in the morphogenesis of the blood vessels in the embryo and may play an important part as an antiangiogenesis factor during maturation and ageing. West and colleagues demonstrated that partial degradation products of hyaluronic acid have the opposite effect, that is, angiogenesis. ${ }^{24}$ Mutations of hyaluronic acid metabolism may have pervasive effects on angiogenesis, which would explain the failure to thrive in patients with HGPS.

Cytogenetic analysis of the postmortem skin biopsy specimens from identical twins showed an inverted insertion of chromosome 1 , described as $46 \mathrm{XY}$, inv ins $(1 ; 1)$ (q32; $444 \mathrm{q} 23)$ in $70 \%$ of the cells. It raises the possibility that

Table 1 Clinical manifestations of HGPS

\begin{tabular}{ll}
\hline Incidence: USA & 1 in 8 million live births \\
Male : female & $1.5: 1$ \\
General features & Short stature, weight low for height, absent sexual maturation \\
Cutaneous features & Loss of subcutaneous fat, wrinkled skin, circumoral cyanosis \\
Head & Craniofacial disproportion, micrognathia, alopecia, prominent scalp veins, prominent eyes, \\
& "plucked bird" appearance \\
Teeth & Abnormal and delayed dentition \\
Trunk & $\begin{array}{l}\text { Pyriform thorax, short dystrophic clavicles } \\
\text { Limbs }\end{array}$ \\
"Horse riding" stance, wide based shuffling gait, coxa valga, thin limbs, stiff joints, \\
Mentation & dystrophic nails, etc \\
Voice & Thinal IQ \\
& Thin and high pitched \\
\hline
\end{tabular}


Table 2 Theories of ageing (modified from ref 22, 27, 36)

\begin{tabular}{ll}
\hline Type of theory & Explanation \\
\hline Genetic-molecular & Codon restriction: accuracy of DNA-mRNA impaired \\
& Error theories: accuracy of mRNA-proteins impaired \\
& Gene regulation: postreproductive changes in gene expression \\
& Somatic mutation: radiation damage to DNA \\
& Wear and tear: with use the body wears out and dies \\
Age pigments: lipofuscin deposit as a primary cause of ageing & Free radicals: lead to cell damage and thus ageing \\
Cellular & enzymes \\
& Wide range of species-species variation in lifespan \\
& Balance between energy investment in reproduction and maintenance \\
& Neuroendocrine control: a "biological clock" controls development through neural and \\
Evolutionary & hormonal mechanisms \\
Disposable soma & Immune control: thymus gland as an "immunological clock" leading to cell destruction due to \\
System level & failure to recognise self \\
& Changes occurring throughout the whole lifespan, often involving deterioration of an \\
anatomical/physiological nature
\end{tabular}

a gene for progeria may be located at the break point site of the inserted chromosome segment. ${ }^{21}$ Subsequent study by Abdenur et al demonstrated the endogenous growth hormone resistance and malnutrition in children with HGPS. ${ }^{25}$ Ted Brown demonstrated that overnight growth hormone concentrations were within the normal range $(8-12 \mu \mathrm{g} / \mathrm{l})$, but their insulin-like growth factor I concentrations in the plasma were very low $(0.1-0.2 \mu \mathrm{U} / \mathrm{l}) .{ }^{21} \mathrm{~A}$ trial of growth hormone treatment resulted in a marked increase in linear growth and a paradoxical drop in basal metabolic rates. Therefore, the failure to thrive in patients with HGPS may be due to a bioinactive form of growth hormone and a lack of vasculogenesis caused by excessive excretion of hyaluronic acid. It now appears that progeria subjects may have bioinactive growth hormone or endogenous growth hormone resistance, which may benefit from growth hormone treatment along with nutritional supplementation. ${ }^{21}$

\section{Pathophysiology of ageing}

Ageing in man results from a complex interaction of genetic and environmental factors. ${ }^{26}{ }^{27}$ Many overlapping and sometimes conflicting theories of ageing exist and the emergence of a unified theory still seems unlikely. Experimental studies of senescence are difficult to design because of the variable effect of diseases and other extrinsic factors.

What is ageing? Ageing is a developmental process, part of the cycle beginning at conception and ending with death. Ageing (physiology) is not the same as disease (pathology); diseases do become much more common in the aged population. ${ }^{28}$ Cells get old and die just like the whole organism, but they do it at a more rapid rate. Normal cells that differentiate stop dividing and eventually die. Cancer cells continue to divide and apparently escape senescence. A fact for which there is currently no definite explanation is that in a variety of different species, a chronically decreased energy intake prolongs life. ${ }^{29}$ However, the cells of the epidermal layer of the skin shed themselves within a few days. The lifespan of the red blood cell is about 120 days. Various theories have been put forward to accommodate the following observations about ageing (listed in table 2).
One theory of ageing holds that tissues age as a result of random mutations in the DNA of somatic cells, with consequent cumulative abnormalities. ${ }^{28}{ }^{29}$ Others hold that cumulative abnormalities are produced by increased cross linkage of collagen and other proteins, possibly due to the non-enzymatic combination of glucose with amino groups on these molecules. A third theory envisions ageing as the cumulative result of damage to the tissues by free radicals formed in them. Species with longer life produce more superoxide dismutase, an enzyme that inactivates oxygen-free radicals. Some investigators have speculated that in mammals there is a biological ageing clock, possibly situated in the hypothalamus, that is responsible for ageing via hormonal or other pathways. $^{29} 30$

\section{Hayflick limit to cell division}

The Hayflick limit is the number of cell divisions that human fibroblasts in a cell culture can undergo before stopping. ${ }^{30-32}$ Human cells can only divide about 50 times when cultured outside the body. ${ }^{30}$ By contrast, many human cancer cells continue to divide unchecked and are called immortal. ${ }^{28}$ The number of divisions is gradually reduced in cells from ageing individuals, including those of a younger chronological age, who have an inherited form of early ageing called Werner's syndrome, HGPS. This has led to the idea that ageing is intracellular. ${ }^{30}$

\section{Premature ageing syndromes (HGPS,} metageria, Down's syndrome)

The very rare inherited premature ageing, or progeria, syndromes lead to early death (due to cardiovascular or cerebrovascular complications) with many of the characteristics of very old people. ${ }^{5283}$ The fibroblast cells of affected people demonstrate a reduced ability to replicate. ${ }^{28}$ Studies of people who age early suggest that there are genes which promote ageing once they are activated, as if "ageing genes" act as a genetic clock. ${ }^{30}$

\section{Cross linkage in DNA, collagen, and proteins}

Increased cross linking between collagen fibres and proteins interferes with connective tissue functions. In DNA, cross links may form that 
are difficult to break, leading to errors in replication. ${ }^{28}$

\section{Autoimmune damage and reduction in immune response}

The occurrence of autoimmune diseases-for example, pernicious anaemia, thyroid disease, vasculitis (connective tissue diseases)-is more common as people get older. Elderly people have impaired protective immune responses and therefore become more prone to infections, such as pneumonia and tuberculosis. ${ }^{28}$ Could the immune system be a "clock" that triggers ageing? ? $^{30}$

\section{Rate of DNA repair linked to life expectancy}

Rate of DNA repair varies from species to species. DNA gets damaged in the rough and tumble of cellular functions, so DNA repair enzymes spontaneously deal with the problems. Species that are long lived, such as humans and elephants, tend to have a better DNA repair system than short lived species. ${ }^{28} 30$ Reduced DNA repair ability has also been proposed in cells from progeria patients. ${ }^{34} \mathrm{DNA}$ helicase uncoils DNA for a variety of replicative, transcriptional and repair processes, with failure resulting in genomic instability. ${ }^{35}$ The occurrence of higher rates of malingnancy in premature ageing syndromes, for example, Werner's syndrome, is likely to be due to chromosomal abnormalities resulting from reduced cell cycle regulation. ${ }^{36}$

\section{Damage theories}

Mitochondrial DNA and the membranes of mitochondria are damaged during a person's life time, probably due to free radicals. ${ }^{30}$ Free radicals are oxidants (electron donor), produced as a byproduct of metabolism, and have an extra electron. They are very unstable, and when they combine with cell membranes this leads to damage producing lipid peroxides and aldehydes, which increase cross linking in proteins and DNA. ${ }^{28}$ Antioxidants, for example, vitamins $\mathrm{C}$ and $\mathrm{E}$ and selenium are regarded as "antiageing" drugs. There is no evidence that these drugs work. The damage and toxic accumulation theories have been described as the "clinker" theory, where toxic metabolites accumulate and damage cells. Somatic mutation caused by radiation damage to DNA is another variant of the error theories, but is now discounted as the levels of radiation would have to be far too high to explain ageing changes. ${ }^{28}$

Programmed ageing and genetic theories Genetic programme theories state that ageing is developmentally controlled and that the whole process from conception through differentiation and growth to senescence is under a regulatory gene complex. ${ }^{28}$ However, it is possible that longevity genes are linked with characteristics that make us more resistant to disease. Programmed ageing is linked to the development and morphogenesis of cells under genetic control. ${ }^{27} 28$
Programmed ageing theories and damage theories can be brought together. It is postulated that the regulation of DNA repair mechanisms, of oxidative stress (free radicals), and of immune mechanisms is under programmed genetic control. There is evidence that the major histocompatability complex in the mouse is associated with both immunological competence and longevity. ${ }^{28}$ Support for the genetic variability in longevity comes from the observation that late egg laying drosophila have a life span that is a mean 20 days more than early laying flies. ${ }^{37} 38$

Progeria syndromes provide further evidence of genetic contribution to ageing, although the effects of these disorders are not clearly the same as ageing in all organs. ${ }^{28}$

\section{Evolutionary theories of ageing}

Evolutionary ageing theories are designed to explain wide range of life spans the from species to species. It was once thought that ageing was an adaptive response to the threat of overcrowding and that it might be associated with accelerated evolutionary change that was essentially "good" for the species. This is absolutely wrong. In the wild average life expectancies are very short compared with survival in a protected environment. ${ }^{28}$ Natural selection (the key evolutionary process) does not operate at a species level but at the level of individuals who survive with a greater chance of preserving their germ line (that is, those cells that are involved in reproduction).

\section{Disposable soma theory}

An organism has to achieve a balance between the energy spent in reproduction and the energy spent in maintenance. The evolutionary fitness of a species depends upon the energy investment in maintenance. If the energy investment in maintenance is very high a species will become immortal, but this is at the expense of evolutionary fitness (that is, the responsiveness to change according to environmental hazards). ${ }^{28}$ By contrast, if the energy investment in maintenance is very low the species age rapidly, and have a short life span with a higher reproductive potential. Species like mice have a very high risk of hazards and must complete their reproduction as quickly as possible. They invest very little energy in maintenance and therefore have a short life span but a higher reproductive rate.

The disposable soma theory therefore states that, for a given level of environmental hazard, the optimal level of maintenance is less than the minimum required for immortality or nonageing. In other words, wear and tear are an inevitable part of being alive. ${ }^{28}$

\section{Age changes theory}

Age related changes occur throughout the whole lifespan, often involving deterioration of an anatomical/physiological nature. ${ }^{22}{ }^{27}$ Many cellular functions appear to change with increasing age. Random molecular damage leading to accumulation of defects, for example, lipofuscin, causes age related changes in 
organs and systems that we recognise as ageing. ${ }^{28}$

The ageing eye reveals many defects, for example, cataract, presbyopia, entropion, senile macular degeneration. Hearing impairment (presbycusis) is extremely common in old age. With increasing age renal size and function decline. More dramatic is the fall in the number of nephrons, half of which are lost between the ages of 40 and 70 years. Similarly ageing brain, heart, lungs, bones, joints, muscles, and even immune systems have impaired function and so on.

Therefore, progeria syndromes do support the hypothesis that at a DNA level, ageing results, in part, from an imbalance between DNA damage and repair. In some of these syndromes, malignant transformations occur due to severe chromosomal aberrations. Genetic theories of ageing and damage theories can be viewed as not being mutually exclusive, but as part of a much more complicated interaction of internal and external factors. ${ }^{27}$ It is possible that there is a network of mechanisms under genetic control that all age at different rates, but probably the mechanisms that have the shortest life are those that determine the average life span of a species. ${ }^{28}$

\section{Pathophysiology of HGPS}

Arteriosclerosis, nephrosclerosis, myocardial fibrosis, and vascular calcifications are significant cardiovascular findings. ${ }^{26}$ Necropsy studies on patients with HGPS revealed gross abnormalities in skin, cardiovascular and cerebrovascular tissues, and bones. ${ }^{9}$ Loss of subcutaneous fat is always a consistent finding. Recently, magnetic resonance angiography demonstrated bilateral occlusion of internal carotid and vertebral artery origins. ${ }^{39}$ Pathological studies have demonstrated premature subintimal fibrosis in the blood vessels. ${ }^{40}$

Skin abnormalities vary according to the site and age of the patient. The epidermis is usually normal to mildly hyperkeratotic, with increased melanin in the basal layer. There is completely disorganised collagen, which is usually thickened and hyalinised. The elastic tissue is surprisingly normal. The density of sweat glands, sebaceous glands, blood vessels, and hair follicles may be normal or reduced. Arrector pilorum muscles are usually prominent.

Skeletal changes are also common. They are short statured. The cranial bones and the diaphysis of the long bones are thin. The clavicles show osteolysis, with replacement by fibrous tissue. Osteoporosis is common. Avascular necrosis of the heads of the femurs and acro-osteolysis of the terminal phallanges are the other dominant bone changes. ${ }^{26}$ Delayed and abnormal dentition are also common.

\footnotetext{
Diagnosis

Diagnosis may be established by the following:

- Characteristic clinical features.

- Classical geriatric disorder of the young.

- Thin, high pitched voice.

- Typical gait and coxa valga.
}

- Radiography of the skull: craniofacial disproportion, delayed and abnormal dentition.

- Radiography of the hands: radiolucent terminal phallanges.

- Urine test: excessive excretion of the glycosaminoglycan, hyaluronic acid.

- Culture of skin fibroblast exhibited $76.1 \%$ DNA repair capacity compared with normal.

- Genetic: sporadic dominant mutation.

- Arterial biopsy: premature atherosclerosis and subintimal fibrosis.

\section{Differential diagnosis}

(1) ACROGERIA

Acrogeria is probably an autosomal dominant condition. The important clinical features consist of cutaneous atrophy of the hands and feet, prominent trunkal veins, easy bruising, proptosis and normal life span. ${ }^{41}$

(2) WERNER'S SYNDROME

Werner's syndrome is an autosomal recessive condition. Clinical features include alopecia with fine hair, dermosclerosis, pigmentation, telangiectasia, "bird-like" facies, stocky trunk, sclerodactyly, cataracts, impaired glucose tolerance test, and increased incidence of malignancies. There is increased excretion of macromolecular acidic glycosaminoglycans. ${ }^{42}$

\section{(3) METAGERIA}

Metageria is most probably an autosomal recessive condition. The clinical features consist of fine and thin fair, cutaneous atrophy, wasting, mottled hyperpigmentation of the skin, "bird-like" facies, tall stature, and diabetes in some cases. ${ }^{43}$

\section{(4) COCKAYNE'S SYNDROME ${ }^{44}$}

Cockayne's syndrome is an autosomal recessive condition. The patients are cachectic dwarfs. They are usually deaf and blind due to optic atrophy. Other features include dry thin hair, wrinkled skin due to loss of subcutaneous fat, sunken eyes and "beak-like" nose. Their gait is usually ataxic due to cerebellar involvement.

\section{(5) ROTHMUND-THOMSON SYNDROME}

Rothmund-Thomson syndrome is an autosomal recessive condition. The clinical features include alopecia with fine hair, poikiloderma (that is, cutaneous atrophy with pigmentation, giving it a mottled appearance), juvenile cataracts, hyperkeratotic plaques, and frontal bossing. There appears to be increased incidence of malignancies - for example, osteosarcoma, squamous cell carcinoma, etc in this condition. ${ }^{45}$

(6) ATAXIA TELANGIECTASIA ${ }^{46}$

Ataxia telangiectasia is an autosomal recessive condition. The clinical features consist of cutaneous atrophy, telangiectasis, cerebellar ataxia, premature canities (loss of pigments in the hair), and, at times, abnormal involuntary movements-for example, chorea, dystonia, etc. The patients are usually immunodeficient. 


\section{Key points:}

- HGPS is a very rare genetic disorder.

- Characteristic facies, wrinkled skin, prominent scalp veins, typical posture and gait, "sculpted nose", and stunted growth will lead to the diagnosis.

- Normal motor and mental development.

- No demonstrable abnormalities of growth hormone, thyroid stimulating hormone, parathyroid hormone, and adrenal hormones.

- Increased insulin resistance.

- Presence of abnormal collagen.

- Varying degrees of generalised atherosclerosis involving chiefly the larger arteries in postmortem studies.

- Although the majority die of cardiovascular or cerebrovascular disease, between 7 and 27 years of age, many other features of ageing are absent. ${ }^{5}$

There may be signs of peripheral neuropathy as well. There appears to be increased incidence of malignancies in this syndrome.

\section{Prognosis}

The prognosis is guarded. These patients suffer from premature vascular events, for example, acute myocardial infarction, ${ }^{47}{ }^{48}$ confirmed by postmortem studies. The other modes of death are due to congestive cardiac failure caused by coronary artery disease ${ }^{32}{ }^{49}$ and also due to cerebral infarction..$^{39}$ In a review of 60 cases, acute myocardial infarction was the most common cause of death, followed by chronic myocardial infarction. ${ }^{5}$ The majority of deaths occurred during the second decade of life. ${ }^{27}$ The maximum life span was noted to be 27 years. ${ }^{52}$

We are very grateful to Pamela Sarkar for her help in typing the manuscript. We are also indebted to Olivia Christophersen, Research Officer, Census, Population and Health Statistics, ONS, UK and Dr Marleen De Smedt, Head of Sector "Health and Safety Statistics", Eurostat, L-2920 LuxembourgKirchberg, for providing statistical data on progeria syndrome (HGPS).

1 Brown WT. Human mutations affecting ageing: a review. Mech Ageing Dev 1979;9:325-6.

2 Hamilton WJ. The biology of the smokey shrew (Sorex fumeus fumeus miller). Zoologica (NY) 1940;23:473-91.

3 Brown WT. Progeria: a human disease model of accelerated ageing. Am f Clin Nutr 1992;55:1222S-4S

4 Hutchinson J. Congenital absence of hair and mammary glands with atrophic condition of the skin and its glands with atrophic condition of the skin and its
appendages in a boy whose mother had been almost totally bald from alopecia areata from the age of six. Medicochirurgical Transactions 1886;69:473-7.

5 DeBusk FL. The Hutchinson-Guilford progeria syndrome. 7 Pediatr 1972;90:697-724.

6 Tonunaga M, Wakamatsu E, Soto K, et al. Hyaluronuria in a case of progeria (Hutchinson-Guilford syndrome). $7 \mathrm{Am}$ Geriatr Soc 1978;26:296-302.

7 Brown WT, Kieras FJ, Houck GE, et al. A comparison of adult and childhood progerias: Werner syndrome and Hutchinson-Guilford progeria syndrome. Adv Exp Med Biol 1985;190:229-44.

8 Guilford H. Progeria: a form of senilism. Practitioner 1904;73:188-217.

9 Badame AJ. Progeria. Arch Dermatol 1989;125:540-4.

10 Martin GM. Genetic modulation of the senescent phenotype in homosapiens. Genome 1989;31:390-7.

11 Balin AD, ed. Contribution of in vitro skin fibroblast studies from individuals with genetic disease that predispose to from individuals with genetic disease that predispose to
accelerated ageing phenomena to our understanding of the ageing process. New York: Raven Press, 1989: 93-119.
12 World Health Organisation. International classification of diseases ninth revision (ICD9). A manual of the International classification of diseases, injuries and causes of death. Geneva: WHO, 1977-78; Vol 1: 154

13 Mills RG, Weiss AS. Does progeria provide the best model of accelerated ageing in humans? Gerontology 1990;36:84-98

14 Moen C. Orthopaedic aspects of progeria. F Bone foint Surg [Am] 1982;64:542-6.

15 Gamble JG. Hip disease in Hutchinson-Guilford progeria syndrome. F Pediatr Orthop 1984;4:585-9.

16 Faragher RG. Cell senescence and human ageing: where's the link? Biochem Soc Trans 2000;28:221-6.

17 Faragher RG, Kipling D. How might replicative senescence contribute to human ageing? Bioessays 1998;20:985-91.

18 Ishikawa F. Ageing clock: the watchmaker's masterpiece. Cell Mol Life Sci 2000;57:698-704.

19 Goyns MH, Lavery WL. Telomerase and mammalian ageing: a critical appraisal. Mech Ageing Dev 2000;114:6977.

20 Allsopp RC, Vaziri H, Patterson C, et al. Telomere length predicts replicative capacity of human fibroblasts. Proc Natl Acad Sci U S A 1992;89:1014-8.

21 Zebrower M, Kieras FJ, Brown WT. Urinary hyaluronic acid elevation in Hutchinson-Guilford progeria syndrome. Mech Ageing Dev 1986;35:39-46.

22 Sweeney KJ, Weiss AS. Hyaluronic acid in progeria and the aged phenotype. Gerontology 1992;38:139-52.

23 Feinberg R, Beebe D. Hyaluronate in vasculogenesis. Science 1983;220:1177-9.

24 West DC, Hampson IN, Arnold F, et al. Angiogenesis induced by degradation products of hyaluronic acid. Science 1985;228:1324-6.

25 Abdenur JE, Brown WT, Freidman S, et al. Endogenous growth hormone resistance and malnutrition in children with Hutchinson-Guilford progeria syndrome (HGP). Pediatr Res 1991;29:73A(abstract).

26 Pesce K, Rothe MJ. The premature ageing syndromes. Clin Dermatol 1996;14:161-70.

27 Dyer CAE, Sinclair AJ. The premature ageing syndromes: insights into the ageing process. Age Ageing 1998;27:73-80.

28 Bennett GCJ, Ebrahim S. The essentials of health care in old age. 2nd Ed. New York: Oxford University Press, 1995: 3-10.

29 Ganong WF. Review of medical physiology. 18th Ed. Stamford, Connecticut: Appleton and Lange, 1997: 43-4.

30 Vellacott J, Side S. Understanding advanced human biology. 1 st Ed. London: Hodder and Stoughton Educational, 1998: 315-9.

31 Hayflick L. Aspects of cellular ageing. Rev Clin Gerontol 1993;3:207-22.

32 Hayflick L. The limited in vitro lifespan of human diploid cell strains. Exp Cell Res 1965;37:614-36.

33 Makous N, Freidman S, Yakovac W, et al. Cardiovascular manifestation in progeria. Report of clinical and pathologic findings in a patient with severe arteriosclerotic heart disease and aortic stenosis. Am Heart f 1962;64:334-46.

34 Wang S, Nishigori C, Yagi T, et al. Reduced DNA repair in progeria cells and effects of gamma-ray irradiation on UV-induced unscheduled DNA synthesis in normal and progeria cells. Mutat Res 1991;256:59-61.

35 Hoehn H, Bryant EM, Au K, et al. Variegated translocational mosaicism in human skin fibroblast cultures. Cytogenet Cell Genet 1975;15:282-98.

36 Tlsty TD, Briot A, Gualberto A, et al. Genomic instability and cancer. Mutat Res 1995;337:1-7.

37 Luckinbill LS, Clare MJ. Selection for lifespan in Drosophila melanogaster. Heredity 1985;55:9-18.

38 Fukui HH, Pletcher SD, Curtsinger JW. Selection for increased longevity in Drosophila melanogaster: a response to Baret and Lints. Gerontology 1995;41:65-8.

39 Smith AS, Wiznitzer M, Karaman BA, et al. MRA detection of vascular occlusion in a child with progeria. Am $\mathcal{F}$ Neuroradiol 1993;14:441-3.

40 Gabr M, Hashem N, Hashem $M$, et al. Progeria: a pathologic study. $\mathcal{F}$ Pediatr 1960;57:70-7.

1 Matsumura Y, Hamanaka H, Okuwa T. A case of acrogeria. f Dermatol 1993;20:572-6.

42 Goto M, Murata K. Urinary excretion of macromolecular acidic glycosaminoglycans in Werner's syndrome. Clin Chem Acta 1978;85:101-6.

43 Greally JM, Boone LY, Lenkey SL, et al. Acrometageria: a spectrum of premature ageing syndromes. Am $\mathcal{F}$ Med Genet 1992;44:334-9.

44 Levinson P, Zimmerman W, Grammet L, et al. Cockayne syndrome. F Comput Assist Tomogr 1982;6:1172-4.

45 Vennos EM, Collins M, James WD. Rothmund-Thomson syndrome: review of the world literature. 7 Am Acad Dermatol 1992;27:750-62.

46 McFarlin DE, Strober W, Waldmann TA. Ataxiatelangiectasia. Medicine 1972;51:281-314.

47 Atkins L. Progeria, report of a case with post-mortem findings. $N$ Engl f Med 1954;250:1065.

48 Talbot NB, Butler AM, Pratt EL, et al. Progeria; clinical, metabolic and pathologic studies on a patient. Am $\mathcal{7}$ Dis Child 1945;69:267

49 Album MM, Hope JW. Progeria, report of a case. Oral Surg 1958;11:985. 